Supporting Information for

\title{
Dual photoisomerization on distinct potential energy surfaces in a UV absorbing rhodopsin
}

Yusaku Hontani, Matthias Broser, Meike Luck, Jörn Weißenborn, Miroslav Kloz, Peter Hegemann and John T.M. Kennis*

*Corresponding author. Email: j.t.m.kennis@vu.nl 

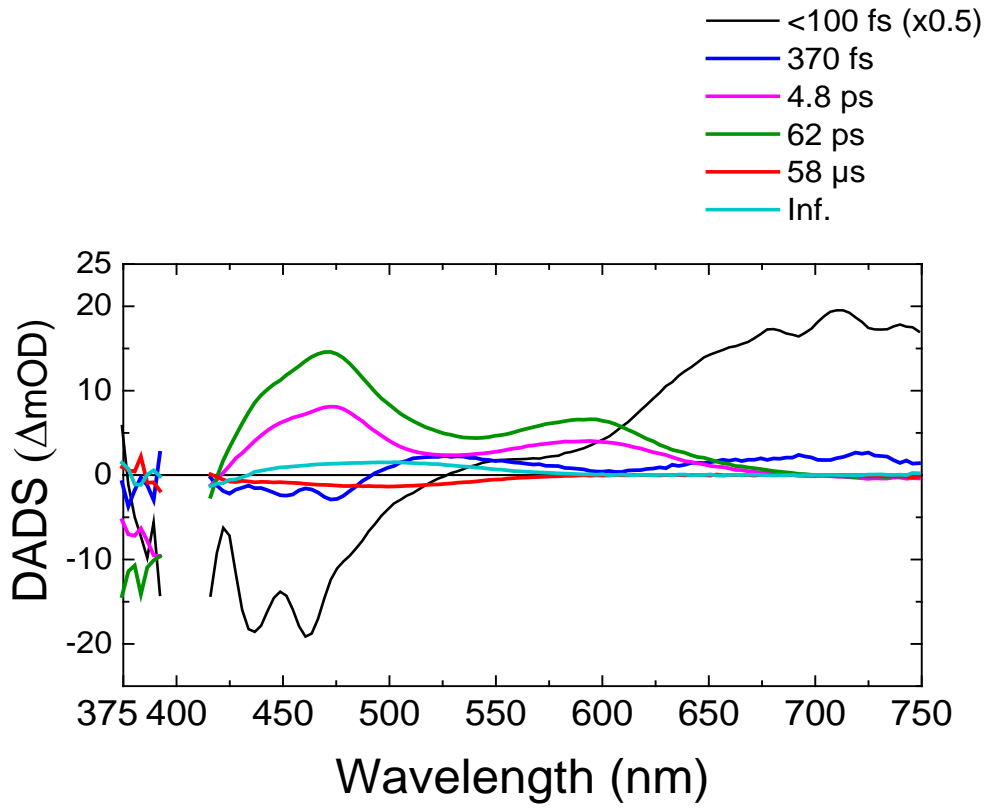

Figure S1. Decay-associated difference spectra (DADS) of transient absorption data of Rh-UV state of HKR1 upon $400 \mu \mathrm{m}$ excitation. The amplitude of the first DADS (black line) is scaled down by 2. 

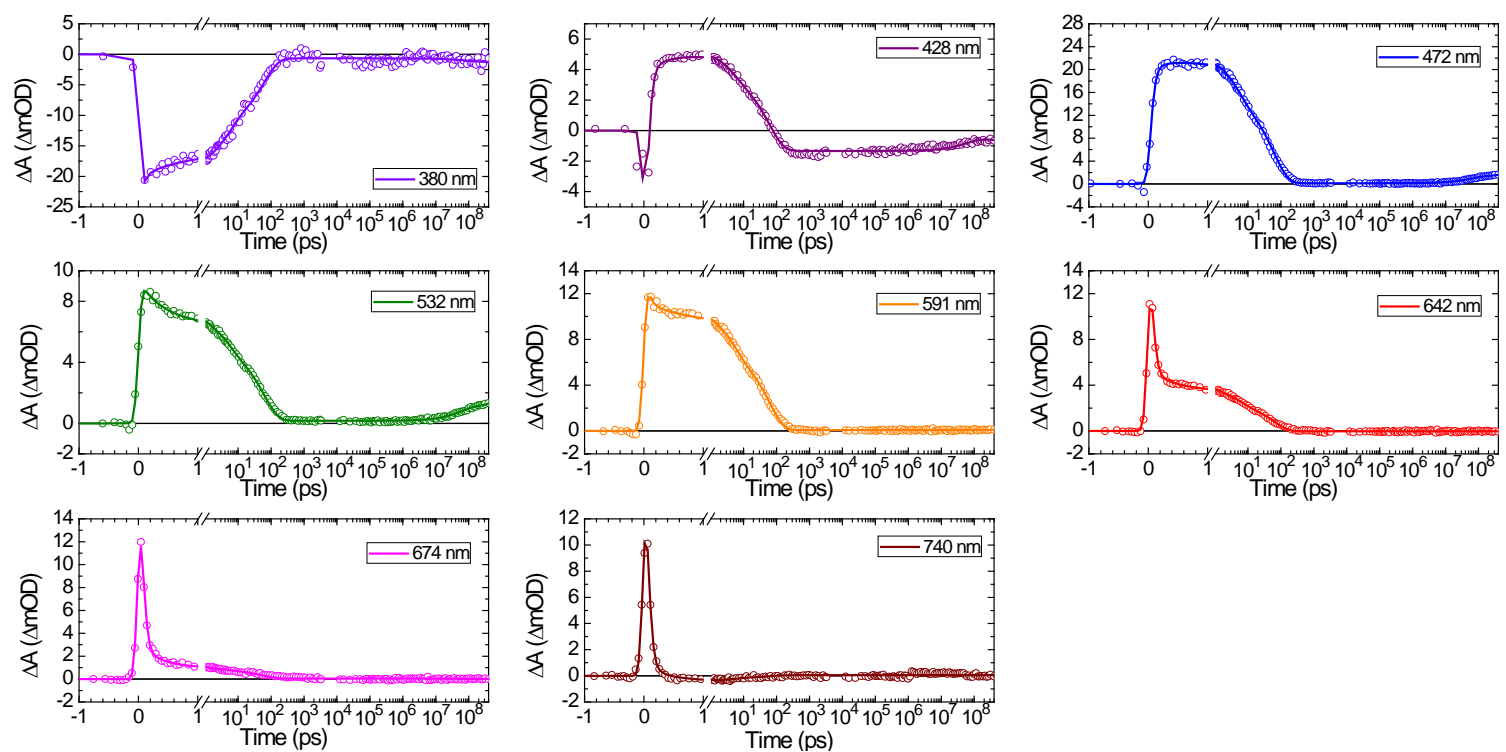

Figure S2. Selected transient absorption time traces with fitting curves. The open dots and solid lines show raw data and fitting curves, respectively. The time axis is linear up to $1 \mathrm{ps}$, and logarithmic thereafter. 
a

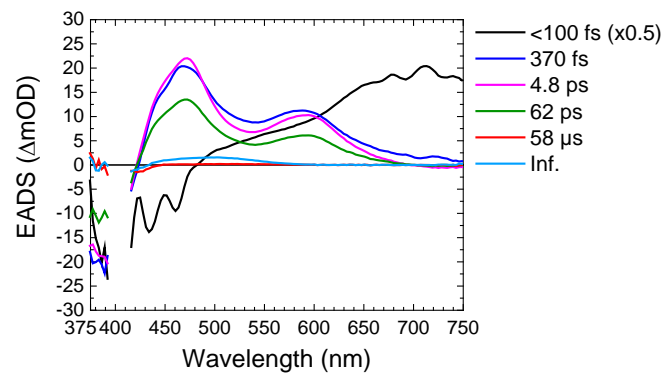

b

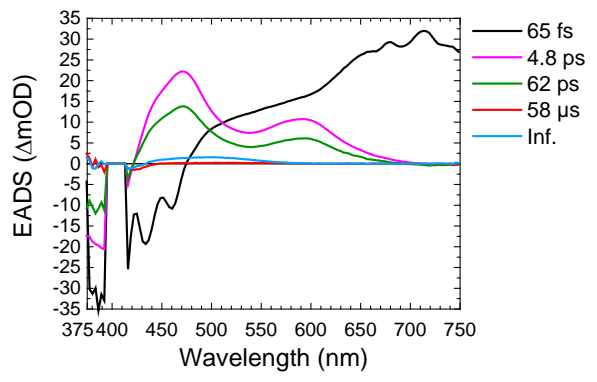

$642 \mathrm{~nm}$
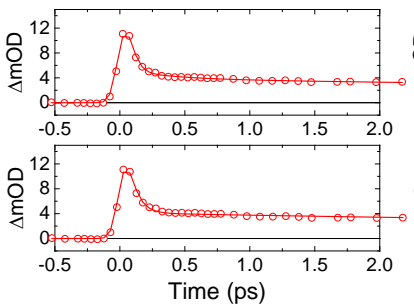

$740 \mathrm{~nm}$
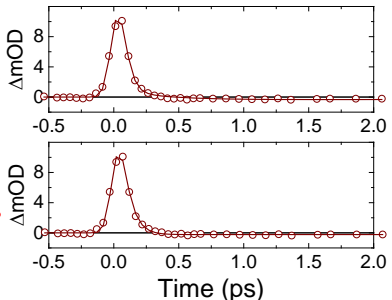

Figure S3. Comparison of global fitting of the transient absorption (TA) spectra with 6 and 5 components. Evolution-associated difference spectra (EADS) fitted (a) with 6 components and (b) with 5 components. (c) Fitted time traces at selected wavelengths up to 2 ps with 6 components (top) and 5 components (bottom). The open dots and solid lines show raw data and fitting curves, respectively. 
a

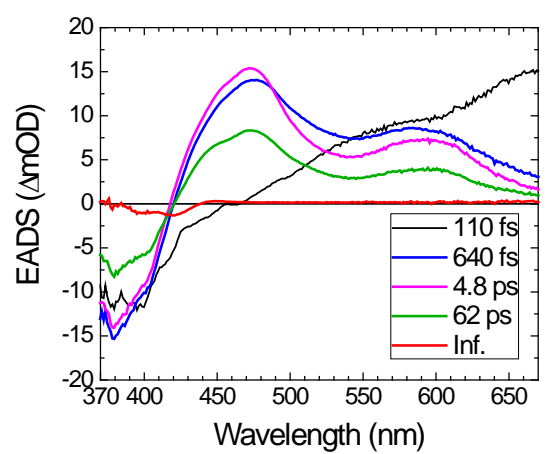

b

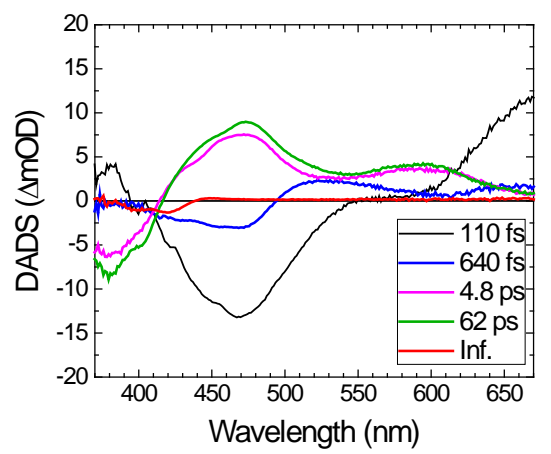

C
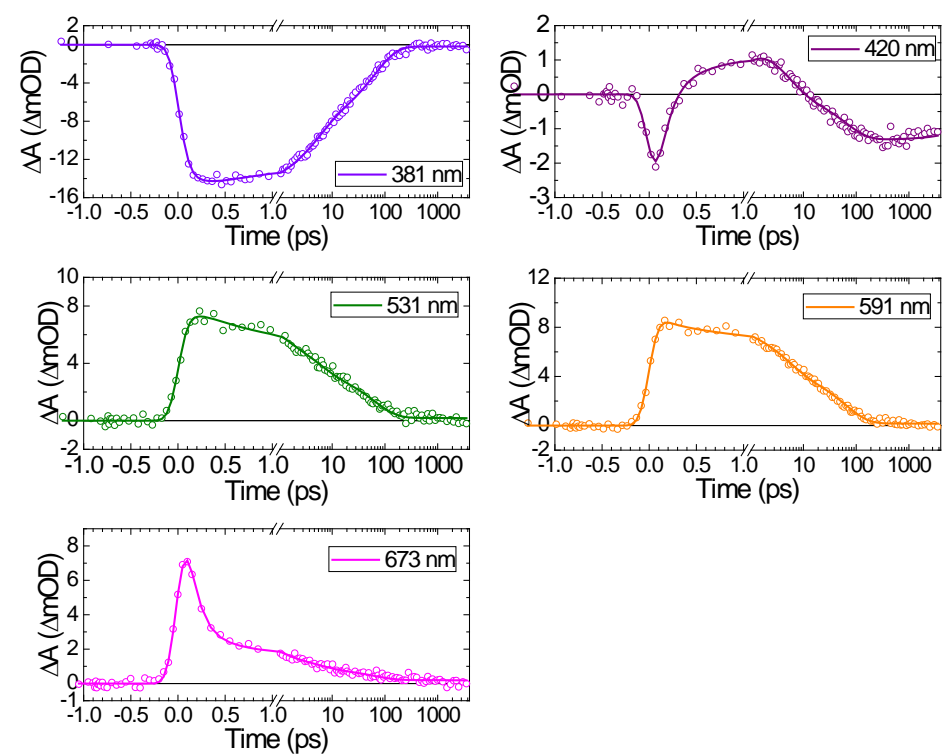
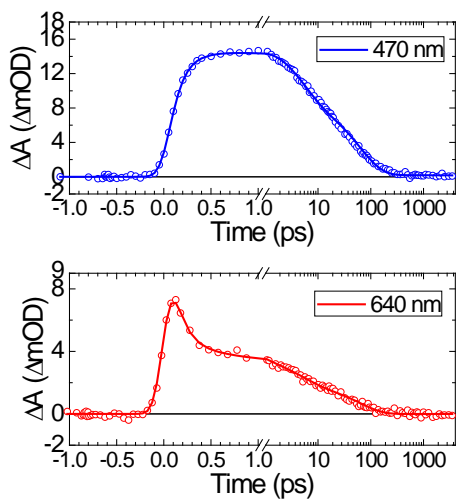

Figure S4. Transient absorption data from Luck et al., $2012^{1}$ refitted with the ultrafast 110 fs fitting component. (a) Evolution-associated difference spectra (EADS) and (b) decay-associated difference spectra (DADS). (c) Time traces at selected wavelengths. The open dots and solid lines show raw data and fitting curves, respectively. 

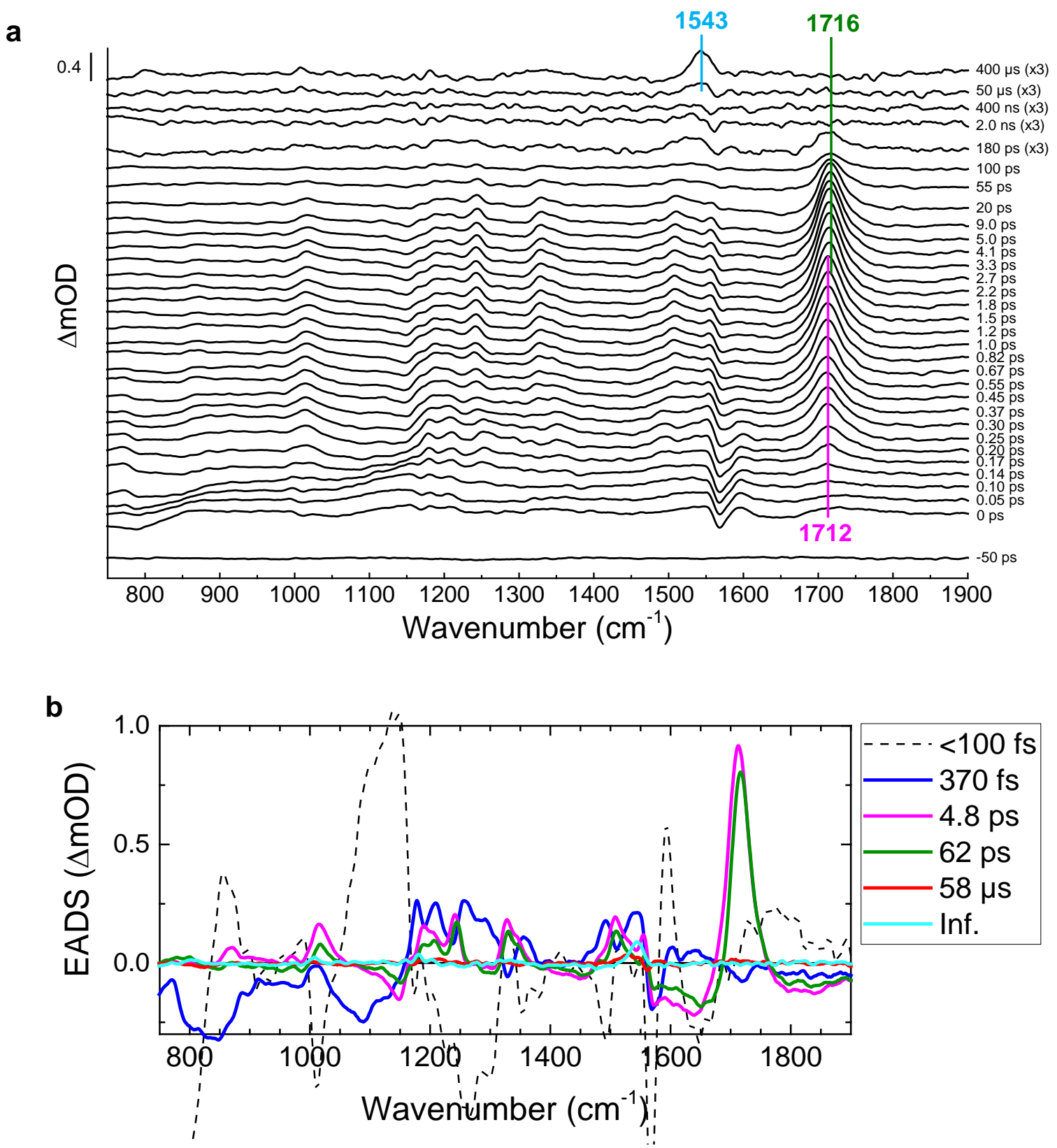

Figure S5. FSRS spectra of Rh-UV of HKR1 upon 400-nm excitation with a preresonant 800-nm Raman pump. (a) Selected raw FSRS spectra. (b) Globally fitted evolution-associated difference spectra (EADS). The same time constants to the TA data were applied to the FSRS global fitting. 

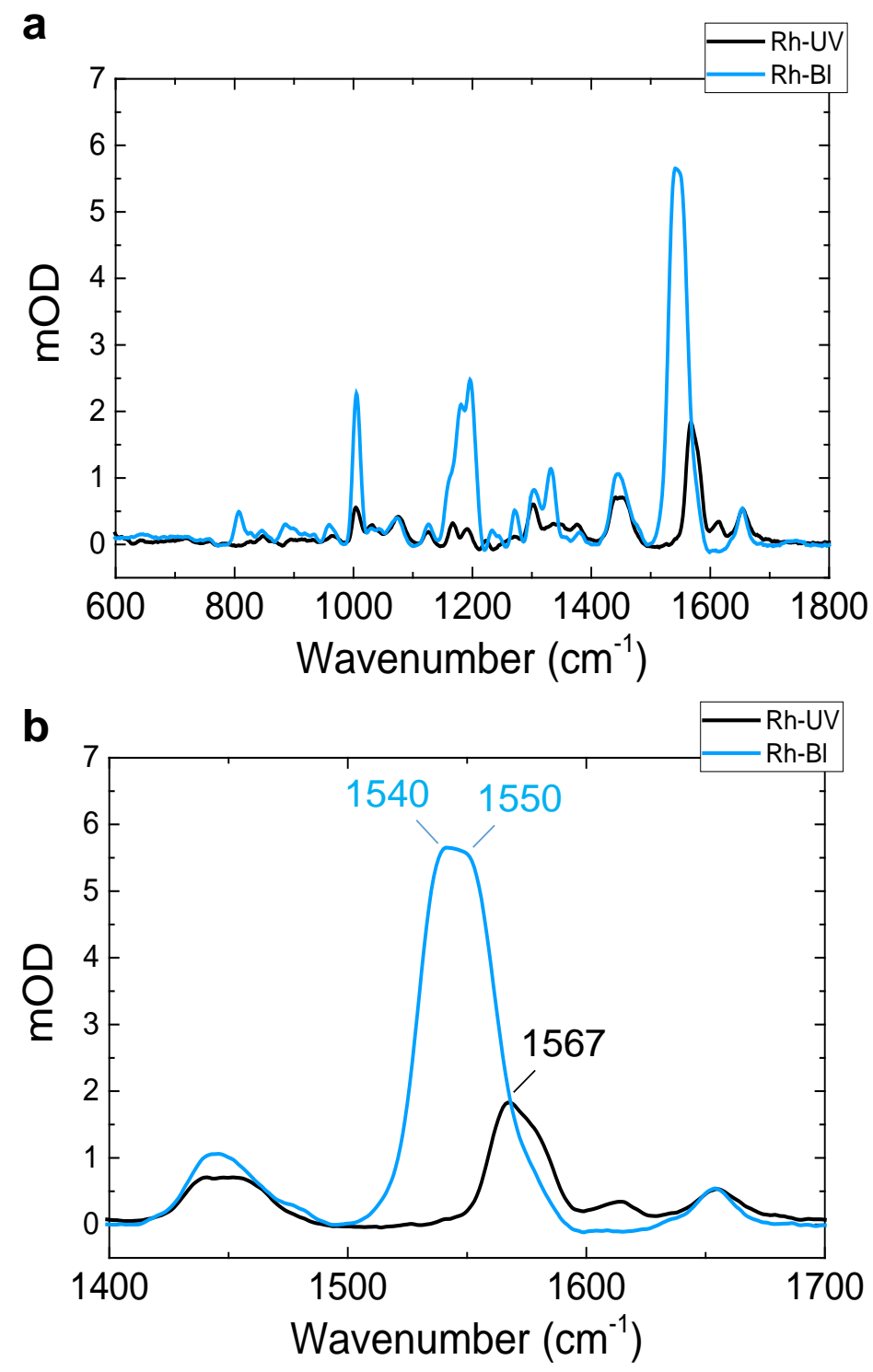

Figure S6. Ground state stimulated Raman spectra of Rh-UV1 (black lines) and Rh-Bl (blue lines) upon preresonant Raman excitation at $800 \mathrm{~nm}$ taken at identical experimental conditions. The Raman spectra of (a) the region $600-1800 \mathrm{~cm}^{-1}$ and (b) the region $1400-1700 \mathrm{~cm}^{-1}$. The vibrational bands coincide with those reported by Luck et al. ${ }^{1}$ The amplitude of Rh-Bl is higher than Rh-UV1 because of increased preresonance with the $800 \mathrm{~nm}$ pump of the former. Rh-UV1 shows a single RSB C=C stretch at 1567 $\mathrm{cm}^{-1}$. In addition, it shows bands at $1650 \mathrm{~cm}^{-1}$ and a shoulder at $1580 \mathrm{~cm}^{-1}$. These were not observed in Luck et al., ${ }^{1}$ and are assigned to protein Amide I and Amide II, respectively, which become apparent due to the preresonant excitation at $800 \mathrm{~nm}$. The $\mathrm{Rh}-\mathrm{Bl}$ state shows double RSB C=C stretch bands at 1540 and $1550 \mathrm{~cm}^{-1}$, as in Luck et al., ${ }^{1}$ along with band at $1650 \mathrm{~cm}^{-1}$ for the RSB C=NH mode and protein Amide I. In addition, it shows a shoulder at $1580 \mathrm{~cm}^{-1}$, assigned to Amide II. 


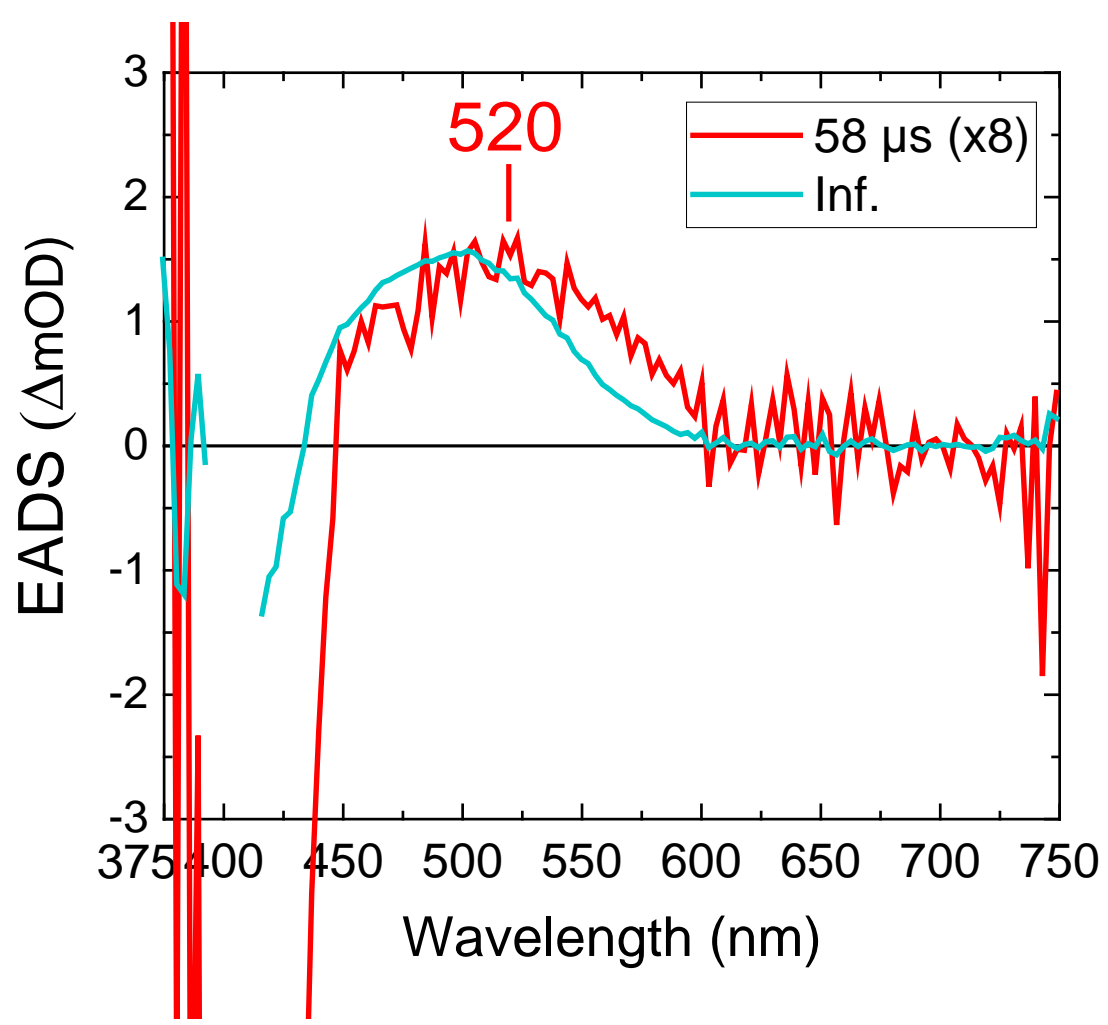

Figure S7. The $58 \mu$ s transient absorption EADS (red line) scaled on the non-decaying EADS (cyan line), demonstrating that this component has a low concentration of protonated retinal species with a maximum absorption at $520 \mathrm{~nm}$. 

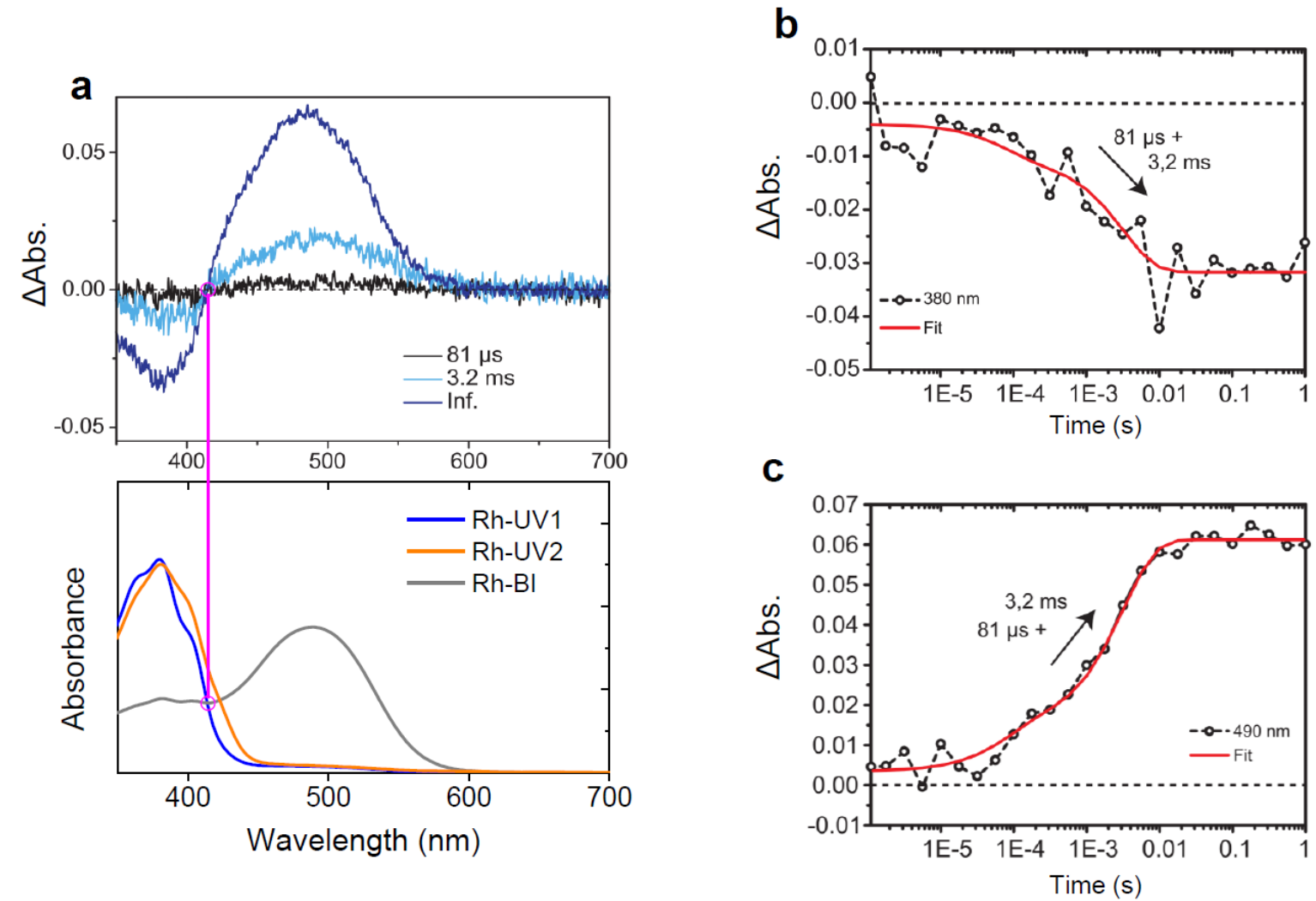

Figure S8. Flash photolysis experiments of Rh-UV1 state of HKR1 upon $380 \mathrm{~nm}$ excitation; data from Luck et al., 2012. ${ }^{1}$ (a) Globally fitted evolution-associated difference spectra (EADS, top panel) and ground-state absorption of Rh-UV1 (blue line), Rh-UV2 (orange line) and Rh-Bl (gray line) species (bottom panel). The Rh-UV1 and Rh-UV2 states were formed under blue ( 470 nm) and orange ( 560 $\mathrm{nm}) \mathrm{LED}$ illumination to the $\mathrm{Rh}-\mathrm{Bl}$ state, respectively. The magenta line lying across the top and bottom panels is inserted to demonstrate that the zero-crossing section of the EADS (top panel) corresponds to the spectral crossing-point of Rh-UV1 and Rh-Bl (bottom panel). Time traces of flash photolysis data at (b) $380 \mathrm{~nm}$ and (c) $490 \mathrm{~nm}$ with fitting curves. The $\Delta$ Absorption ratio at $\sim 500 \mathrm{~nm}$ of over the $3.2 \mathrm{~ms}$ component (cyan) the infinite component (dark blue) is $\sim 0.31$ (top panel, Figure S8a). On the other hand, in Figure S7, the $\Delta$ Absorption ratio at $\sim 500 \mathrm{~nm}$ of the $58 \mu$ s component (red, Figure S7) over the infinite component (cyan, Figure S7) is $\sim 0.13$. Since the cyan lines in Figures S7 and S8a indicate the same kinetic component, the $\Delta$ Absorption ratio at $\sim 500 \mathrm{~nm}$ of the $58 \mu$ s component (red, Figure S7) over the final non-decaying component (dark blue, top panel, Figure S8a) is $\sim 0.04$. Therefore, it can be estimated that the fast-growing $\sim 500$-nm absorbing state consists of only $\sim 4 \%$ of the total protonated product yield. 

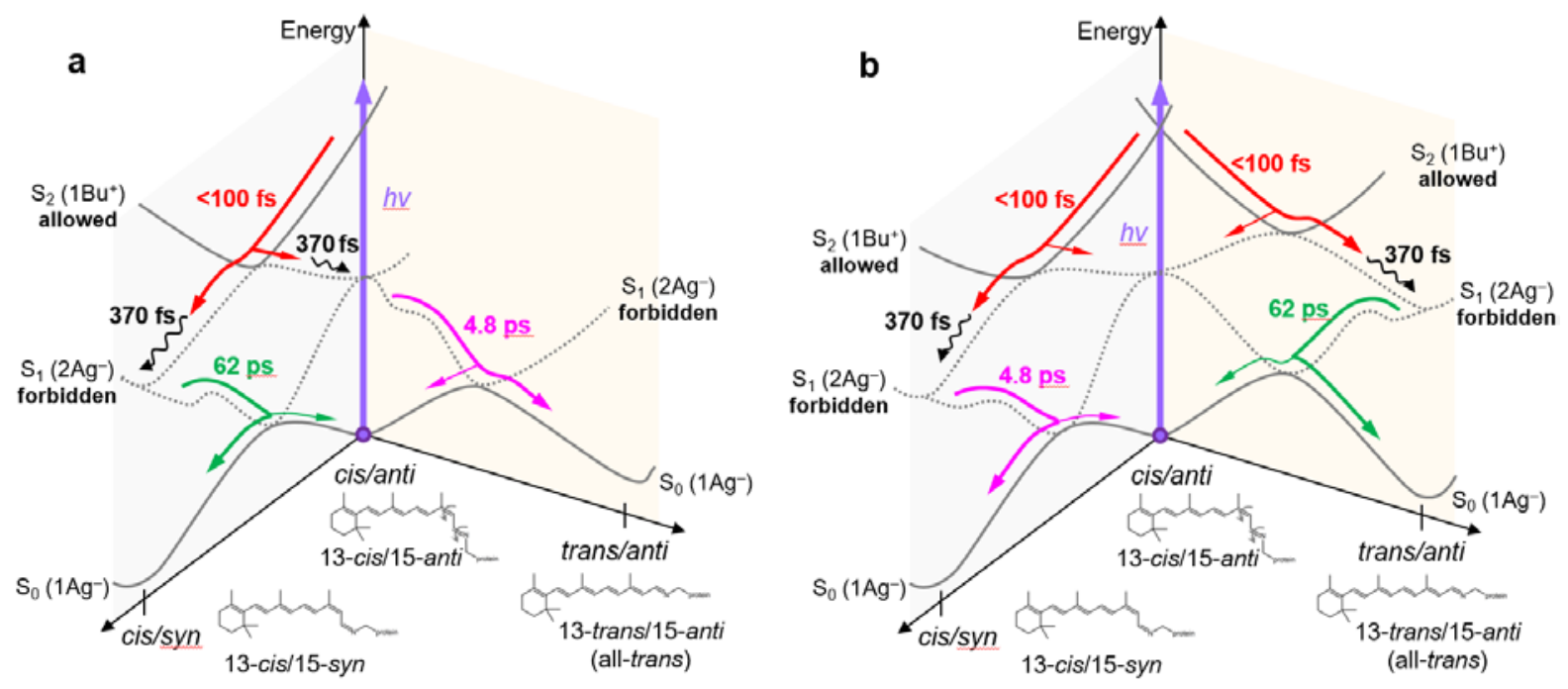

Figure S9. Alternative excited-state reaction models of Rh-UV HKR1. The red arrow indicates the isomerization pathway(s) on the $\mathrm{S}_{2}-\mathrm{S}_{1}$ surfaces. 


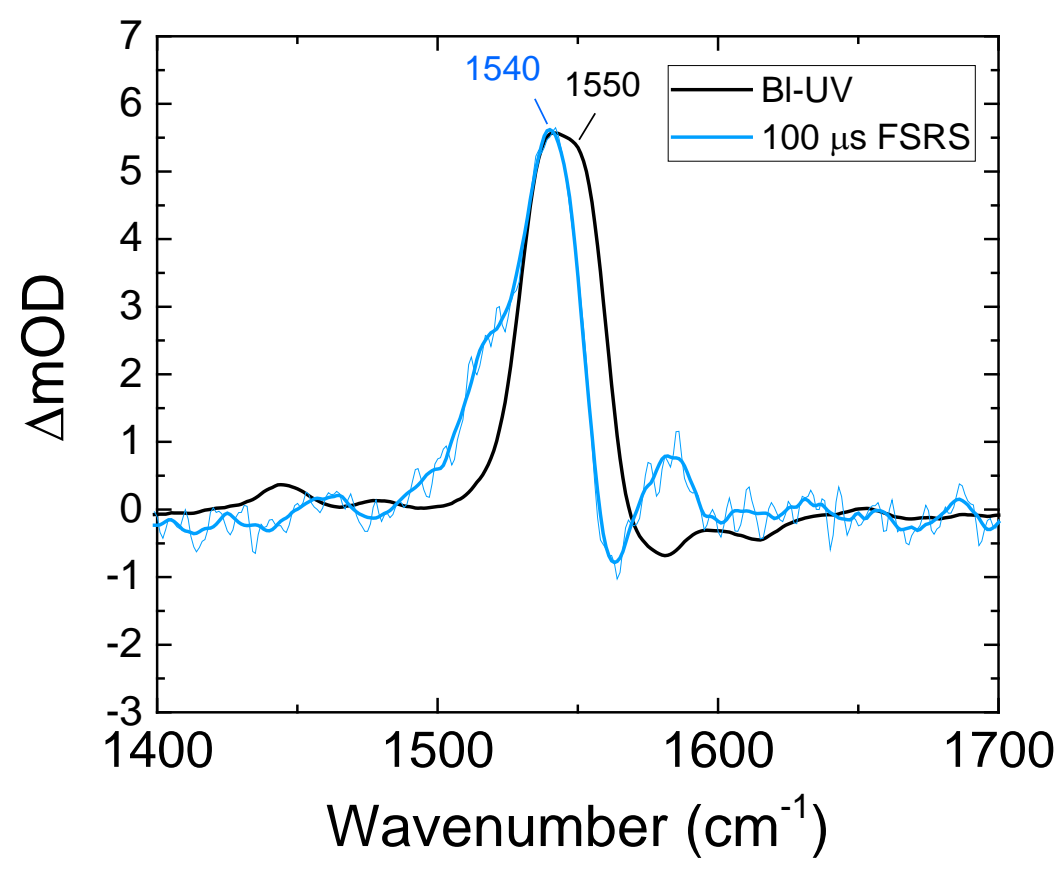

Figure S10. The $100 \mu$ s FSS spectrum (data from Figure 4c) scaled on the steady-state Rh-Bl minus Rh-UV stimulated Raman spectrum (reconstructed from Figure S6) taken on the same setup. 


\section{References}

$1 \quad$ M. Luck, T. Mathes, S. Bruun, R. Fudim, R. Hagedorn, T. M. T. Nguyen, S. Kateriya, J. T. M. Kennis, P. Hildebrandt and P. Hegemann, J. Biol. Chem., 2012, 287, 40083-40090. 